\title{
OPTIMIZATION OF AZIMUTHAL SCAN PROCEDURE FOR ABSOLUTE STRUCTURE DETERMINATION
}

\author{
J. Grochowski AND P. SERda \\ Regional Laboratory of Physicochemical Analysis and Structural Research \\ Jagiellonian University \\ Ingardena 3, 30-060 Kraków, Poland
}

\begin{abstract}
The paper describes the application of the azimuthal scan technique for absolute structure determination using X-ray anomalous dispersion. A diffraction pattern with rotation around the scattering vector provides information about various anisotropic effects: absorption, extinction and multi-beam interaction. Recording of azimuthal scans for Friedel pairs is proposed as an alternative to single reflection measurement. Several factors affecting the smoothness of the azimuthal scan curves are discussed and a procedure for eliminating various sources of errors, involving a Fourier filtering, is proposed in order to increase feasibility of chiral discrimination.
\end{abstract}

PACS numbers: $61.10 . \mathrm{Nz}, 33.15 . \mathrm{Bh}$

\section{Introduction}

Azimuthal scan ( $\psi$-scan) consists of a rotation of the crystal sample around the diffraction vector and recording of the resulting intensity variations as a function of the rotation angle $(\psi)$. For the purpose of absorption correction in $\mathrm{X}$-ray diffraction experiments it was first proposed by Furnas in 1957 [1]. This author proposed an approximation, which limited the application of his method to low-absorption crystal samples (characterized by low value of the product of the linear absorption coefficient and sample size).

The method was applied by North et al. [2] and further developed for the purpose of macromolecular crystallography by Kopfmann and Huber [3] and Huber and Kopfmann [4]. The first attempt to apply the method in small molecule crystallography and precision single crystal X-ray diffractometry was made by Katayama and Sakabe [5] and Flack [6]. Flack's method employs also recording of azimuthal scans for several reflections including symmetry-equivalent, not imposing any limitation of low absorption condition on measured crystals, which makes the method particularly useful for highly symmetric (higher than monoclinic) inorganic substances. A comprehensive review of empirical absorption correction origin 
and development was given by Bartels [7]. The $\psi$-scan absorption technique as well as its modification allowing absorption-extinction correction [8] offered convenient automatization providing a list of absorption corrections as a function of diffractometer setting angles $(\omega, \theta, \phi, \chi)[9]$, and was implemented in contemporary crystallographic software (Molen, XTAL, GSAS).

\section{Factors influencing azimuthal scan shapes}

\subsection{Extinction and absorption}

The shape of azimuthal scan curves recorded for several reflections against the background of a calculated azimuthal absorption correction curves shows amplified variations which are either co-periodical or have a completely different variation pattern $[10,11]$. For example, detailed analysis of the shape of the azimuthal scan curves for a spherical sample of ammonium bitartrate crystal indicated that the shape of the curve, however periodical with $180^{\circ}$ period of the $\psi$ angle, is quite far from the expected smoothness. The observed variation of the integrated intensity for different azimuthal angles may be attributed to anisotropic extinction effects $[12,13]$. Denne [10] reports the observation of significant anisotropic extinction effects for $\alpha$-glycine crystals. The occurrence of this extinction effect was correlated with reflection intensity, most pronounced for low-angle highest intensity reflections. Zachariasen [14] proposed two idealized models for anisotropic extinction. For type I, anisotropy of the mosaic spread parameter $(\kappa)$ is not the same for all azimuthal angles and characteristic $g$ parameter is inversely proportional to the mosaic spread, and type II anisotropy - where extinction is caused by domain asphericity. For most of organic crystals the first type of anisotropic extinction is more probable.

\subsection{Mosaic spread}

With decreasing perfection of the organic crystal an occurrence of extinction as a factor affecting azimuthal curve will diminish. A typical mapping of reflection $(\omega-\theta$ plot) for an organic substance [15] reveals for several reflections significant mosaic anisotropy. In extreme case, both anisotropic mosaicity and split reflection patterns are observed [16] seriously affecting azimuthal scan shapes. The situation is critical with low-divergence synchrotron radiation beams.

\subsection{Scan direction, sample illumination}

In practice, azimuthal scan is a sequence of integral intensity measurements of a given Bragg reflection for various discrete values of the $\psi$ angle. A single intensity measurement requires another scanning procedure (usually in the $\omega-\theta$ direction or $\omega$ in the case of synchrotron beam) providing a minimum box of enclosure encapsulating a reciprocal lattice node of finite size. Ob viously, our measuring direction will depend on the instrumental conditions, particularly the primary beam divergence, spectral composition and Bragg angle. For precise intensity measurements uniform illumination of the crystal sample in various orientations is essential. It requires proper choice of the sample size with respect to primary 
beam homogeneity region. The crystal mount should be made of low absorption support (fibre glass, carbon fibre rod, etc.) taking into consideration that screening effect may cause a non-uniform illumination of the sample for various orientations (stick-and-glue effect). A detailed discussion of this effect in protein crystallography was given by Krieger et al. [17] and by the present authors for the purpose of anomalous scattering measurements [18].

\subsection{Positioning errors}

Positioning errors also affect - especially reproducibility of - the azimuthal scan curve. Each goniostat which has less than five active circles provides azimuthal rotation by a combined rotation about several axes. In order to minimize the resulting positioning errors, special pre-orientation of the crystal sample is necessary. For precise measurements, dedicated six-circle goniostats are constructed and used with rotation anode tube or synchrotron radiation sources.

\subsection{Umweganregung}

Since intensity variations described above have angular width of a few up to a few dozens degrees, they can be easily distinguished from intensity variation implied by multi-beam interaction appearing within very small angular $\psi$ range. Detailed discussion of the multi-beam interactions appearing on azimuthal scan curves was carried out by Rossmanith and coworkers [19, 20]. The UMWEG95 program developed by Rossmanith [21] allows - starting from wavelength, the unit cell parameters and orientation matrix of the crystal sample - to localize regions of possible multi-beam interactions. With known structure, also an estimation of the Umweganregung and Aufhellung peaks intensity is possible.

\section{Absolute structure determination}

Absolute structure determination represents the most serious task in the field of naturally originated compounds composed of light atoms $(\mathrm{C}, \mathrm{H}, \mathrm{O})$ only. Most of these compounds are homochiral. C, N, O are weak chirality markers in anomalous dispersion diffraction experiments since their absorption edges are quite far from the range of wavelengths used for structure determination.

Absolute structure determination is based on measuring differences in intensities of Friedel pairs, i.e., reflections with $h k l$ and $-h-k-l$ Miller indices, which are caused by anomalous dispersion. Historically, such differences/inequalities are called Bijvoet differences/inequalities. The idea to compare whole azimuthal scan curves rather than intensities measured at one position (typically $\psi=0$, i.e. $\omega=\theta$, bisecting position) was developed in order to avoid accidental results of Bijvoet inequalities caused by random errors and to avoid azimuthal scan range where systematic errors could make the comparison unreliable. Recording of azimuthal scan for the determination of absolute structure is carried out for selected Friedel pairs in order to detect significant differences of two curves [22], a voiding regions of azimuthal angles, where systematic errors are particularly severe.

Using the model of refined crystal structure, most dispersion-sensitive Friedel pairs of reflections (omitting those biased with extinction) are selected and examined for sufficient and uniform illumination of the sample by the primary beam 
within the chosen $\psi$ range. Friedel mates with their $\chi$ values differing by more thàn $15^{\circ}$ are rejected.

For the crystal mounting, special low absorption $0.01 \mathrm{~mm}$ thick glass capillaries are employed in order to minimize the stick-and-glue effect. An appropriate azimuthal angle range is selected free of multi-beam interaction regions using the UMWEG95 program. Next, careful mapping of diffraction spots is carried out $(\omega-\theta$ plot, OTPLOT) [23] and an optimum scan direction in reciprocal space is chosen. If necessary, the reflection width and profile are changed using primary beam monochromators with different mosaic spread (graphite, germanium) and varying tube take-off angle.

In case when intensities of the Friedel mates are on the edge of intensity measuring precision, additional zero-difference Friedel pairs are recorded as a reference. When morphology allows for an optical sample pre-orientation, special crystal settings are prepared for several samples in order to diminish positioning errors with 4-circle diffractometer. In the first setting, so-called "top reflection", the $\psi$ and $\phi$ axes coincide; $\psi$-scan recording is then effectuated by a single $\phi$-axis rotation. Such a setting allows one to conduct the alignment procedure of the whole diffractometer directly using the measured Friedel pair. However, it requires a capillary mount (instead of standard stick and glue) in order to provide symmetric sample illumination. An alternative setting corresponds to $\psi$-axis parallel to $\chi$-axis, allowing for a $\psi$-scan by a single $\chi$ rotation. The latter setting is applicable only on the Eulerian cradle goniometer, especially with a $\phi$-axis offset.

The use of synchrotron radiation source offers several advantages for recording weak dispersion phenomena:

- due to low spatial divergence and high energetic resolution, the recording of high orders of interference is enhanced, allowing for an increase in the anomalous phase rotation ratio $k=\delta f^{\prime \prime} /\left(f_{0}+\delta f^{\prime}\right)$ without increasing the wavelength; on the other hand, such low divergence beam is much more discriminating with respect to mosaic inhomogeneity;

- high brilliance decreases exposure time and makes it possible to decrease the sample size to nearly sub-extinction sizes, without a significant loss in counting statistics.

Interpretation of experimental results strongly depends on the symmetry of the crystal sample and we can distinguish two categories:

I. The crystal sample cross-section with the diffraction plane has at least 2-fold symmetry: then all systematic errors, such as extinction, absorption, multi-beam interactions, attributed to the crystal sample should compensate. The measured azimuthal scan curves are "parallel" and their similarity is only disturbed by random errors from positioning and counting statistics.

II. The crystal cross-section with diffraction plane is asymmetric: the course of azimuthal scans of both Friedel mates is unrelated to each other.

In the first case, if recorded scan curves fluctuate preserving approximately constant difference, after comparing standard deviations, conclusions can be drawn about Bijvoet inequalities. In case of very weak anomalous scattering effect occurring e.g. when nitrogen is the heaviest atom in the crystal sample, the scan curves usually require filtering procedure using Fourier transform. Since only the dif- 
ference is important, only the lowest frequency is used for Fourier synthesis. As a reference, azimuthal scans are recorded for a Friedel pair with vanishing dispersion component (so-called centrosymmetric reflections).

\section{Experimental}

Diffraction data were collected for cubic BN (borazone), space group $F-43 m$, $a=3.6158(2) \AA$. The sample of $0.1 \mathrm{~mm}$ in size with well-developed faces of
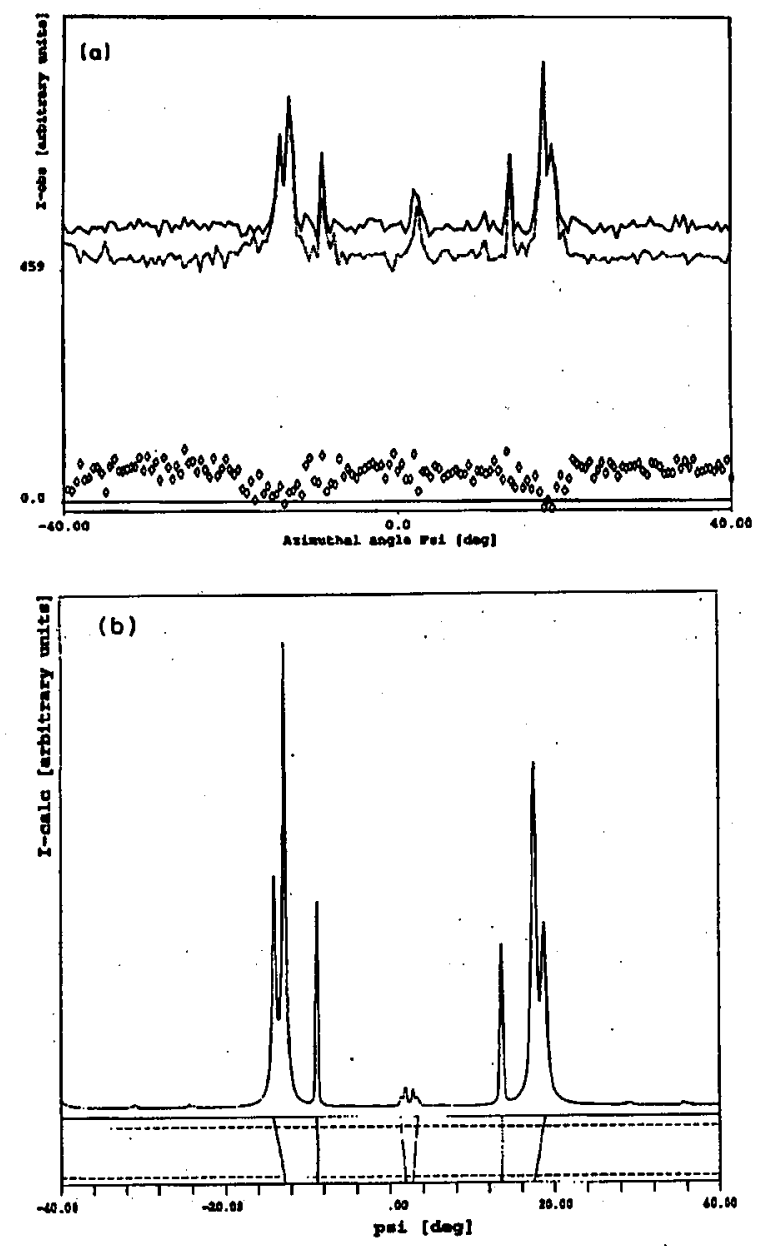

Fig. 1. (a) $\psi$-scan curves collected for borazone for (222) Bragg reflection and its Friedel mate. (b) Multi-beam interaction pattern for (222) reflection in borazone calculated with UMWEG95 program for $\mathrm{Cu}$ radiation. The strongest Umweg events are plotted in the $\psi-\lambda$ diagram. 
positive and negative tetrahedron fell into the first category. The most sensitive Friedel pairs under multipole model refined against $0.5 \AA$ synchrotron data $\left(0.24<\sin \theta / \lambda<1.71 \AA^{-1}\right)$ are $222,-2-2-2$, and their rotational equivalents. The Bijvoet ratio - i.e. Bijvoet difference divided by average intensity of the Friedel pair - for these reflections reaches 0.03 for copper radiation, as calculated from the structural model. The location of maxima for inverted $\psi$-coordinate of the Friedel mate coincide well and the observed fluctuations from flat curves are in good agreement with calculated multi-beam interaction pattern (Fig. 1b), which appears to be the main source of systematic errors for the recorded $\psi$-range. A comparison of Bijvoet differences calculated from the structural model with those determined experimentally shows good agreement (Figs. 1a, 1b). The confidence level increases when the regions of multi-beam interactions are rejected.

The sensitivity of the method for assigning absolute polarity appears higher than the use of Rogers coefficient, a cumulative enantiomer correctness indicator,
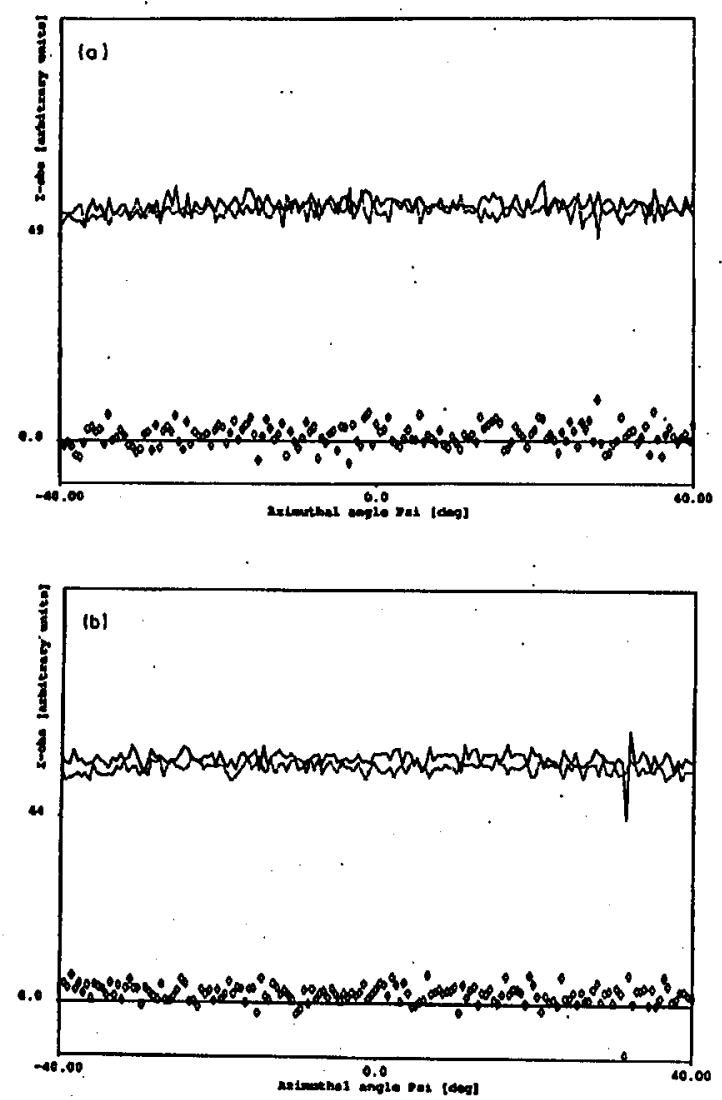

Fig. 2. (a) $\psi$-scani curves recorded with $\mathrm{Cu} K_{\alpha}$ radiation for C39 $\mathrm{O} 8 \mathrm{H} 52$ spirane, for (321) Bragg reflection and its Friedel opposite. (b) $\psi$-scan profiles for spirane, (321) Friedel pair, recorded with Co $K_{\alpha}$ radiation. 
TABLE

A comparison of calculatei Bijvoet ratio values with those estimated from azimuthal scan profiles by statistical treatment and Fourier filtering. The error of Fourier filtering as estimated from series truncation was replaced by a more rigorous statistical estimate. The values of calculated Bijvoet ratio values are model dependent and accurate numerical agreement with experimental values is not always possible.

\begin{tabular}{|c|c|c|c|c|}
\hline \multirow[t]{2}{*}{$\begin{array}{l}\text { Compound } \\
\text { /Friedel pair }\end{array}$} & \multirow{2}{*}{$\begin{array}{c}\text { Radiation } \\
\text { /Wavelength } \\
{[\AA]}\end{array}$} & \multirow{2}{*}{$\begin{array}{c}\text { Calculated } \\
\text { Bijvoet ratio } \\
\Delta I / I\end{array}$} & \multicolumn{2}{|c|}{$\begin{array}{c}\text { Observed } \\
\text { Bijvoet ratio }\end{array}$} \\
\hline & & & $\begin{array}{l}\text { (statistical } \\
\text { treatment) }\end{array}$ & $\begin{array}{l}\text { (Fourier } \\
\text { filtering) }\end{array}$ \\
\hline $\begin{array}{l}\text { Borazone } \\
(-22-2) /(2-22)\end{array}$ & $\begin{array}{c}\mathrm{Cu} \\
1.54178\end{array}$ & 0.035 & $0.036(3)$ & $0.037(3)$ \\
\hline $\begin{array}{l}\text { Borazone } \\
(200) /(-200)\end{array}$ & $\begin{array}{c}\text { Co } \\
1.7902\end{array}$ & 0.000 & $-0.001(2)$ & $0.001(2)$ \\
\hline $\begin{array}{l}\text { Spiran } \\
(3-2-1) /(-321)\end{array}$ & $\begin{array}{c}\mathrm{Cu} \\
1.54178\end{array}$ & 0.014 & $0.017(5)$ & $0.017(5)$ \\
\hline $\begin{array}{l}\text { Spiran } \\
(3-2-1) /(-321)\end{array}$ & $\begin{array}{c}\text { Co } \\
1.7902\end{array}$ & 0.019 & $0.041(5)$ & $0.043(5)$ \\
\hline $\begin{array}{l}\text { Borazone } \\
(-2-22) /(22-2)\end{array}$ & $\begin{array}{c}\text { Co } \\
1.7902\end{array}$ & not available & $0.065(3)$ & $0.065(3)$ \\
\hline
\end{tabular}

which converged to 1.05 (1.71) for $0.5 \AA$ wavelength data. The use of longer wavelength $(0.988 \AA)$ did not increase the confidence level of the Rogers coefficient.

In the second case, physically corresponding to an asymmetric crystal, a crystal sample asymmetrically mounted in a capillary, or a predominant stick-and-glue effect, a more complicated procedure has to be employed. An empirical absorption surface is calculated using reflections with vanishing dispersion components and is used $r$. $r$ absorption correction of appropriate azimuthal curves recorded for Friedel pairs prior to smoothing procedure.

Experimental results were collected for an irregularly shaped crystal sample of C39 O8 H52, revealing large non-uniform mosaicity when exposed to synchrotron radiation beam $(8.5 \mathrm{keV})$. For comparison a Friedel pair of weak reflections (321 and $-3-2-1$ ) with Bijvoet ratio 0.03 was chosen. The corresponding azimuthal scans (Fig. 2a) were recorded using graphite monochromated copper radiation for symmetric $\psi$-range $(-40,40)$ with $0.5^{\circ}$ step size. Each intensity was recorded with $\omega$ scan chosen after analysis of reflection shapes. As could be expected for this case, azimuthal scans of Friedel mates are not congruent. Similar results were obtained for rotation equivalents of the measured pair. The calculated Bijvoet difference from the structural model agreed with the measured one. The experiment repeated for the same crystal with Co radiation indicated that Bijvoet difference increases, as expected, due to larger dispersion correction whereas recordings for 
centrosymmetric reflections (with zero-dispersion component) gave zero Bijvoet difference with high confidence level (Table).

In contrast to BN case, random errors seem to be predominant and Fourier filtering gave fair estimate of the Bijvoet difference confirming the sign of the Bijvoet inequality. Obtained results confirm previously determined absolute structure [15] giving more consistent indication of the correct enantiomer.

The work was supported by the Committee for Scientific Research grant No. PB 1279/P4/93/04. The authors are grateful to Prof. E. F.sssmanith for making the program UMWEG95 available.

\section{References}

[1] T.C. Furnas, Single Crystal Orienter Instruction Manual, General Electric Company, Milwaukee 1957.

[2] A.C.T. North, D.C. Phillips, F.S. Mathews, Acta Crystallogr. A 24, 351 (1968).

[3] G. Kopfmann, R. Huber, Acta Crystallogr. A 24, 348 (1968).

[4] R. Huber, G. Kopfmann, Acta Crystallogr. A 25, 143 (1969).

[5] C. Katayama, N. Sakabe, K. Sakabe, Acla Crystallogr. A 28, 293 (1972).

[6] H.D. Flack, Acta Crystallogr. A 30, 569 (1974).

[7] K. Bartels, in: The Rotation Method in Crystallography, Eds. U.W. Arndt, A.J. Wonacott, North-Holland, Amsterdam 1977, p. 153.

[8] H.D. Flack, Acta Crystallogr. A 33, 890 (1977).

[9] B.A. Frenz \&Associates Inc., SDP. Structure Determination Package, College Station, Texas, USA, and Enraf-Nonius, Delft, The Netherlands 1985.

[10] W.A. Denne, Acla Crystallogr. A 28, 192 (1972).

[11] F.R. Thornley, R.J. Nelmes, Acta Crystallogr. A 30, 748 (1974).

[12] P. Coppens, W.C. Hamilton, Acta Crystallogr. A 26, 71 (1970).

[13] P. Seiler, J.D. Dunitz, Acta Crystallogr. A 34, 329 (1978).

[14] W.H. Zachariasen, Acta Crystallogr. 23, 558 (1967).

[15] J. Grochowski, M. Rutkowska, B. Rys, P. Serda, G. Snatzke, Chem. Ber. 125, 1837 (1992).

[16] J. Grochowski, P. Serda, Z. Dauter, A. Kolasa, Z. Krist. 211, 622 (1996).

[17] M. Krieger, J.L. Chambers, G.G. Christoph, R.M. Stroud, B.L. Trus, Acta Crystallogr. $A$ 30, 740 (1974).

[18] J. Grochowski, P. Serda, Int. Conf. on Anomalous Scaltering, Malente/Hamburg (Germany) 1992, Book of Abstracts, P6.

[19] E. Rossmanith, K. Bengel, Acta Crystallogr. A 51, 134 (1995).

[20] E. Rossmanith, G. Adiwidjaja, J. Eck, G. Kumpat, G. Ulrich, J. Appl. Cryst. 27, 510 (1995).

[21] E. Rossmanith, UMWEG95 - A Program for Calculation and Graphical Representation of Umweganregung Patterns, University of Hamburg, Germany 1995.

[22] J. Grochowski, P. Serda, Acta Phys. Pol. A 82, 147 (1992).

[23] J.D. Schagen, L. Strouver, F. van Meurs, G. Williams, CAD4 version 5.0, 1989. 